\title{
Innovative data mining approaches for outcome prediction of trauma patients
}

\author{
Eleni-Maria Theodoraki ${ }^{1}$, Stylianos Katsaragakis ${ }^{2}$, Christos Koukouvinos ${ }^{3}$, Christina Parpoula ${ }^{3}$ \\ ${ }^{1}$ Department of Statistics and Actuarial-Financial Mathematics, University of the Aegean, Samos Island, Greece; \\ ${ }^{2}$ First Propaedeutic Surgery Clinic, Hippocratio Hospital, Athens, Greece; \\ ${ }^{3}$ Department of Mathematics, National Technical University of Athens, Athens, Greece. \\ Email: parpoula.ch@gmail.com
}

Received 7 June 2010; revised 17 June 2010; accepted 23 June 2010.

\begin{abstract}
Trauma is the most common cause of death to young people and many of these deaths are preventable [1]. The prediction of trauma patients outcome was a difficult problem to investigate till present times. In this study, prediction models are built and their capabilities to accurately predict the mortality are assessed. The analysis includes a comparison of data mining techniques using classification, clustering and association algorithms. Data were collected by Hellenic Trauma and Emergency Surgery Society from 30 Greek hospitals. Dataset contains records of 8544 patients suffering from severe injuries collected from the year 2005 to 2006. Factors include patients' demographic elements and several other variables registered from the time and place of accident until the hospital treatment and final outcome. Using this analysis the obtained results are compared in terms of sensitivity, specificity, positive predictive value and negative predictive value and the ROC curve depicts these methods performance.
\end{abstract}

Keywords: Data Mining; Medical Data; Decision Trees; Classification Rules; Association Rules; Clusters; Confusion Matrix; ROC

\section{INTRODUCTION}

One of the most common and rapidly growing causes of death and disability worldwide, regardless of each country's development level, is traumatic injury [2]. Every day 16,000 people die [3] and trauma is the leading cause of death in the age of 44 years [4] and the fourth leading cause of all ages after cardiovascular, neoplastic, and respiratory diseases. In 1996 the National Academy of Sciences and National Research Council published a report which characterized the injury as the "neglected disease of the modern world".
Due to technological advancements in healthcare domain, an enormous amount of data has been collected over the last few years. This fact is followed by clinician's willingness to explore different technologies and methodologies to analyze these data because their assessment may lead to trends and patterns within the data previously unknown which could significantly enhance their understanding of disease management. Interest in developing prognostic models for binary outcomes has emerged as an essential tool for evaluation of medical treatment. Multiple models exist to assist with prediction of the outcome of injured patients and many comparisons between different methods exist [4]. Traditionally, researchers have used the regression techniques which are not ideal in handling multidimensional, complex biologic data stored in large databases and are time consuming. Therefore, due to the fact that there is no consensus as to an optimal method, it is interesting to explore different methods.

Data mining methods were developed to overcome these limitations. With these techniques, a priori knowledge of variable associations is unnecessary. In contrast to an a priori approach to the selection of predictor variables, data mining allows the discovery of previously unknown variable relationships by exploring a wide range of possible predictor variables. The process of data mining is to find hidden patterns and associations in the data. The utility of data mining methods to derive medical prognostic models from retrospective data, can contribute to increased availability and volume of medical data gathered through systematic use of laboratory, clinical and hospital information systems. Also, it can lead to construction of interpretable prognostic models, handling of noise and missing values, and discovery and incorporation of non-linear patterns and feature combinations.

This paper investigates the utility of machine learning techniques to construct outcome prediction models 
for severe trauma patients and examines measures that will improve the quality of treatment and therefore survivability of patient through optimal management. The study is organized as follows. Section 2 introduces the dataset that was used to investigate the plausibility of modeling the outcome. Statistical methods used for that purpose were classification, association and clustering algorithms. The results of data analysis, and their evaluation according to their predictive ability are reported in Section 3. Section 4 summarizes the results and provides conclusion of the paper.

\section{MATERIALS AND METHODS}

\subsection{Patient Population and Variables}

Our database consisted of cases collected during the project, entitled "Report of the epidemiology and management of trauma in Greece", which was initiated in October 2005 and lasted for twelve months. Study included patients from a range of 30 teaching, and general hospitals who were admitted with a primary diagnosis of injury. Information was gathered for these trauma patients admitted for at least one day in hospital. To avoid biasing estimates, persons who arrived dead or died at the Emergency Room of each hospital were excluded from the analysis. The data and injury scoring was performed by a highly-trained coordinator.

Input variables which were extracted and included to the models concerned demographics, mechanism of injury, month of admission to hospital, whether the patient was referred from another hospital, prehospital care, hospital care and procedures, and outcomes at discharge. Various injury severity scores were also considered including Injury Severity Score (ISS) [5], Abbreviated Injury Scores (AIS) [6], and the Glasgow Coma Score (GCS) [7]. For all models, there was a single output variable: probability of death.

Trauma registry was followed by extensive correction and verification of the data. During preprocessing analysis missing data were also handled. Despite the challenges inherent when data are missing, information could be gained when a thoughtful and systematic analytical approach is used [8]. For that purpose Multiple imputation (MI) was an appropriate method that was used to handle Missing At Random Data in our dataset [9] in order to minimize bias and increase the validity of findings. In this method, multiple $(\mathrm{m})$ versions (typical range 5-20) of the data set are created using available data to predict missing values. These data sets are then used to conduct $m$ analyses, which are then combined into one inferential analysis. The particular appeal of this method is that once completed data sets have been developed, standard statistical methods can be used. Adjusting multiple imputation issues to data mining meth- ods, derived datasets were compared in terms of performance (correctly classified datasets) and the one with the most correctly classified training and test sets was chosen.

The analysis was carried out using the SPSS 17.0 and SPSS Clementine 12.0 statistical software.

\subsection{Data Mining Algorithms}

In this section, we present the data mining methods that were applied to analyze the trauma data. These methods may be categorized according to their goal as feature selection methods, decision tree learners, binary classifier comparison metrics, clustering algorithms and generalized rule induction algorithms.

\subsubsection{Feature Selection}

In order to reduce data set size, minimize the computational time and improve model accuracy, a set of variables selection criteria may be used. Such criteria are the maximum percentage of records in a single category criterion, as fields which have too many records falling into the same category may be omitted and the maximum number of categories as a percentage of records criterion, as if a high percentage of the categories contains only a single case, the field may be ignored. There are two more variable selection criteria, the minimum standard deviation criterion and the minimum coefficient of variation criterion. According to these, fields with standard deviation or respectively coefficient of variance less than or equal to the specified minimum measure may be of limited use. The coefficient of variance is defined as the ratio of the predictor standard deviation to the predictor mean.

A common technique used in data mining is ranking the attributes based on the measure of importance which is defined as (1-p), where $\mathrm{p}$ is the p-value of a chosen statistical test such as the Pearson's chi-square statistic, the Likelihood-ratio chi-square statistic, the Cramer's V or Lambda statistic. More details can be found among others in [10-12] and [13]. The Pearson's chi-square statistical test, is a test of independence between $X$, where $\mathrm{X}$ is a predictor with I categories, and $\mathrm{Y}$, where $\mathrm{Y}$ is the target value with $J$ categories, that involves the difference between the observed and the expected frequencies. The expected cell frequencies under the null hypothesis of independence are estimated by $\hat{N}_{i j}=\frac{N_{i .} N_{. j}}{N}$, where $N$ is the total number of cases, $N_{i j}$ is the number of cases with $\mathrm{X}=i$ and $\mathrm{Y}=j, N_{i}$, is the number of cases with $\mathrm{X}=i\left(N_{i .}=\sum_{j=1}^{J} N_{i j}\right)$ and $N_{. j}$ is the number of cases with $\mathrm{Y}=j\left(N_{. j}=\sum_{i=1}^{I} N_{i j}\right)$. 
Under the null hypothesis, Pearson's chi-square converges asymptotically to a chi-square distribution $x_{d}{ }^{2}$ with degrees of freedom $d=(I-1)(J-1)$. Now, the p-value based on Pearson's chi-square $X^{2}$ is calculated by p-value $=\operatorname{Prob}\left(x^{2}{ }_{d} \succ X^{2}\right)$, where

$$
X^{2}=\sum_{i=1}^{I} \sum_{j=1}^{J}\left(N_{i j}-\hat{N}_{i j}\right)^{2} / \hat{N}_{i j} .
$$

\subsubsection{Decision Trees}

Decision tree models are a structural description which gives the opportunity to develop classification models that may be used to predict or classify future data sets, according to a number of provided decision rules. Future data with unknown classification may be classified just by routing down the tree according to the tests in nodes and assigning the class of the reached leaf. Some of the advantages of this approach are that it is easy understandable, can be transformed into a set of rules (if-then rules) that interpret the data set and finally that the provided tree includes only the important attributes that really contribute to the decisions making. The Classification and Regression Tree (C\&RT) is a method based on recursive partitioning to split the training set into subsets so as to obtain more homogeneous subsets than in the previous step. The split is based on the reduction in an impurity index, and in this study we used the Gini index. CHAID algorithm, or Chi-square Automatic Interaction Detection, is based on the significance level of a statistical test and is a non-binary tree method, that is, it can produce more than two categories at any particular level in the tree. C5.0 algorithm works for data sets where the target field is categorical and builds decision tree by splitting the sample based on the field that provides the maximum information gain at each level.

\subsubsection{Clustering}

Clustering is concerned with grouping records with respect to similarity of values for a set of input fields without the profit of prior knowledge about the form and the characteristics of the groups.

$\mathrm{K}$-means is an iterative algorithm which tries to discover $\mathrm{k}$ clusters, where $(\mathrm{k})$ is defined by the user, so that records within a cluster are similar to each other and distinct from records in other clusters. There are different distance measures, such as Euclidean distance, Manhattan distance and Mahalanobis distance, but in our application we used the Euclidean distance.

The TwoStep cluster method is a scalable cluster analysis algorithm designed to handle very large data sets and both continuous and categorical variables or attributes. It requires only one data pass. It has two steps 1) pre-cluster the cases (or records) into many small sub-clusters 2) cluster the sub-clusters resulting from pre-cluster step into the desired number of clusters. The TwoStep algorithm uses an hierarchical clustering method in the second step to assess multiple cluster solutions and automatically determine the optimal number of clusters for the input data. To determine the number of clusters automatically, TwoStep uses a two-stage procedure that works well with the hierarchical clustering method. In the first stage, the BIC (distance measure) for each number of clusters within a specified range is calculated and used to find the initial estimate for the number of clusters. TwoStep can use the hierarchical clustering method in the second step to assess multiple cluster solutions and automatically determine the optimal number of clusters for the input data.

\subsubsection{Association Rules}

Association rule mining finds interesting associations and/or correlation relationships among large set of data items. Association rules show attribute value conditions that occur frequently together in a given data set. A typical and widely-used example of association rule mining is Market Basket Analysis. Association rules provide information of this type in the form of if-then statements. These rules are computed from the data and, unlike the if-then rules of logic, association rules are probabilistic in nature. In association analysis the antecedent and consequent are sets of items (called itemsets) that are disjoint (do not have any items in common). In addition to the antecedent (the if part) and the consequent (the then part), an association rule has two numbers that express the degree of uncertainty about the rule. The first number is called the support for the rule. The support is simply the number of transactions that include all items in the antecedent and consequent parts of the rule. (The support is sometimes expressed as a percentage of the total number of records in the database). The other number is known as the confidence of the rule. Confidence is the ratio of the number of transactions that include all items in the consequent as well as the antecedent (namely, the support) to the number of transactions that include all items in the antecedent. Clementine uses Christian Borgelt's Apriori implementation. Unfortunately, the Apriori [14] algorithm is not well equipped to handle numeric attributes unless it is discretized during preprocessing. Of course, discretization can lead to a loss of information, so if the analyst has numerical inputs and prefers not to discretize them, may choose to apply an alternative method for mining association rules: GRI.

The GRI methodology can handle either categorical or numerical variables as inputs, but still requires categorical variables as outputs. Rather than using frequent item sets, GRI applies an information-theoretic approach to determine the interestingness of a candidate association 
rule using the quantitative measure $J$. GRI uses this quantitative measure $J$ to calculate how interesting a rule may be and uses bounds on the possible values this measure may take to constrain the rule search space. Briefly, the $J$ measure maximizes the simplicity, goodness-of-fit trade-off by utilizing an information theoretic based cross-entropy calculation. Once a rule is entered in the table, it is examined to determine whether there is any potential benefit to specializing the rule, or adding more conditions to the antecedent of the rule. Each specialized rule is evaluated by testing its $J$ value against those of other rules in the table with the same outcome, and if its value exceeds the smallest $J$ value from those rules, the specialized rule replaces that minimum- $J$ rule in the table. Whenever a specialized rule is added to the table, it is tested to see if further specialization is warranted, and if so, such specialization is performed and this process proceeds recursively. The association rules in GRI take the form If $\mathrm{X}=\mathrm{x}$ then $\mathrm{Y}=\mathrm{y}$ where $\mathrm{X}$ and $\mathrm{Y}$ are two fields (attributes) and $\mathrm{x}$ and $\mathrm{y}$ are values for those fields. The advantage of association rule algorithm over a decision tree algorithm is that associations can exist between any of the attributes. A decision tree algorithm will build rules with only a single conclusion, whereas association algorithms attempt to find many rules, each of which may have a different conclusion. The disadvantage of association algorithms is that they are trying to find patterns within a potentially very large search space and, hence, can require much more time to run than a decision tree algorithm.

\subsubsection{Model Performance}

After categorizing the features and inducing outcome prediction models, different statistical measures can be used to estimate the quality of derived models. In present study discrimination and calibration were calculated. The discriminatory power of the model (Classification accuracy (CA)) measures the proportion of correctly classified test examples, therefore the ability to correctly classify survivors and nonsurvivors. In addition, models were assessed for performance by calculating the Receiver-Operating-Characteristic (ROC) curves, constructed by plotting true-positive fraction versus the false-positive fraction and comparing the areas under the curves. Sensitivity and specificity measure the model's ability to "recognize" the patients of a certain group. If we decide to observe the surviving patients, sensitivity is a probability that a patient who has survived is also classified as surviving, and specificity is a probability that a not-surviving patient is classified as not-surviving. The Area under ROC curve (AUC) is based on a non-parametric statistical sign test and estimates a probability that for a pair of patients of which one has survived and the other has not, the surviving patient is given a greater probability of survival. This probability was estimated from the test data using relative frequencies. A ROC of 1 implies perfect discrimination, whereas a ROC of 0.5 is equivalent to a random model. The above metrics and statistics were assessed through stratified ten-fold cross-validation [15]. This technique randomly splits the dataset into 10 subgroups, each containing a similar distribution for the outcome variable, reserving one subgroup (10\%) as an independent test sample, while the nine remaining subgroups (90\%) are combined for use as a learning sample. This cross-validation process continues until each $10 \%$ subgroup has been held in reserve one time as a test sample. The results of the 10 mini-test samples are then combined to form error rates for trees of each possible size; these error rates are applied to the tree based on the entire learning sample, yielding reliable estimates of the independent predictive accuracy of the tree. The prediction performance on the test data using cross-validation shows the best estimates of the misclassification rates that would occur if the classification tree were to be applied to new data, assuming that the new data were drawn from the same distribution as the learning data. Misclassification rates are a reflection of undertriage and overtriage, while correct classification of injured patients according to their need for TC or NTC care reflects sensitivity and specificity, respectively. Of the two misclassification errors, undertriage is more serious because of the potential for preventable deaths, whereas overtriage unnecessarily consumes economic and human resources.

Given a classifier and an instance, there are four possible outcomes. If the instance is positive (P) and it is classified as positive, it is counted as a true positive (TP); if it is classified as negative $(\mathrm{N})$, it is counted as a false negative (FP). If the instance is negative and it is classified as negative, it is counted as a true negative (TN); if it is classified as positive, it is counted as a false positive (FP).

Given a classifier and a set of instances (the test set), a two-by-two confusion matrix (also called a contingency table) can be constructed representing the dispositions of the set of instances. A confusion matrix contains information about actual and predicted classifications done by a classification system. Performance of such systems is commonly evaluated using the data in the matrix. This matrix forms the basis for many common metrics. We will present this confusion matrix and equations of several common metrics that can be calculated from it for each training, validation and test set in our study. The numbers along the major diagonal represent the correct decisions made, and the numbers of this diagonal represent the errors, the confusion, between the various classes.

The common metrics of the classifier and the additional terms associated with ROC curves such as 
- $\quad$ Sensitivity $=\mathrm{TP} /(\mathrm{TP}+\mathrm{FN})$

- $\quad$ Specificity $=\mathrm{TN} /(\mathrm{FP}+\mathrm{TN})$

- $\quad$ Positive predictive value $=\mathrm{TP} /(\mathrm{TP}+\mathrm{FP})$

- $\quad$ Negative Predictive value $=\mathrm{TN} /(\mathrm{FN}+\mathrm{TN})$

- $\quad$ Accuracy $=(\mathrm{TP}+\mathrm{TN}) /(\mathrm{TP}+\mathrm{FP}+\mathrm{FN}+\mathrm{TN})$

are also calculated for each training, test and validation set.

\section{RESULTS}

Altogether, 8544 patients were recorded with $1.5 \%$ mortality rate (128 intrahospital deaths). The models were therefore trained with a dataset heavily favoured towards survivor. For each of them the binary response variable y (death: 1, otherwise: 0 ) is reported. There were approximately 780.000 data points (92 covariates, 8544 cases). In order to reduce the dimension of the problem we followed the procedure of feature selection, to execute and detect the most statistically significant of them, according to Pearson's chi-square. The final data set which is used for further analysis, included all of the 8544 available patients and the 36 selected factors (fields for data mining). The data set was divided randomly into three subsets: the training set, containing $50 \%$ of cases (t.i 4272), the test set, containing $25 \%$ of cases (2136) and validation set with $25 \%$ of cases (2136). After medical advice, all of the factors were treated equally during the data mining approach, meaning that there was no factor that should be always maintained in the model. Defining maximum percentage of records in a single category equal to $90 \%$, maximum number of categories as a percentage of records equal to $95 \%$, minimum coefficient of variation equal to 0.1 and minimum standard deviation equal to 0.0 , we removed some factors of low importance. Moreover, applying the Pearson's chi-square statistic with respect to the categorical type of the target field and significance level $\mathrm{a}=5 \%$, we finally identified the 36 important variables displayed in C. Koukouvinos webpage, http://www.math.ntua.gr/ ckoukouv.

There were no clear results from C\&RT algorithm because it could not generate a rule set (condition too complex). The summary of C5.0 and CHAID model's predictive ability measured by percentages of correct classified records is displayed in Table 1. The percentage of records for which the outcome is correctly predicted, represents the overall accuracy of the examined method.

Tables 2, 3 and 4 display the confusion matrix for each set for C5.0 algorithm.

The metrics for the training set were: Sensitivity (84.6\%), Specificity (98.4\%), Positive predictive value (45.8\%), Negative Predictive value (99.8\%), Accuracy (98.9\%).

The metrics for the test set were: Sensitivity (72.7\%), Specificity (98.9\%), Positive predictive value (26.6\%), Negative Predictive value (99.8\%), Accuracy (98.83\%).

The metrics for the validation set were: Sensitivity
(64.28\%), Specificity (99.2\%), Positive predictive value (36\%), Negative Predictive value (99.76\%), Accuracy (98.97\%).

The C5.0 tree may be converted into set of rules which are listed in Table 5. In each rule assigned the major classification of the corresponding node. Ruleset for 0 (life) contains 4 rules and ruleset for 1 (death) contains 3 rules.

Table 1. C5.0 and CHAID model's predictive ability.

\begin{tabular}{cccc}
\hline \multirow{2}{*}{ Algorithm } & \multicolumn{3}{c}{ Correctly classified } \\
\cline { 2 - 4 } C5.0 & Training set & Test set & Validation set \\
CHAID & $98.94 \%$ & $98.84 \%$ & $98.97 \%$ \\
\hline
\end{tabular}

Table 2. The confusion matrix for the training set for C5.0 algorithm.

\begin{tabular}{lcc}
\hline \multirow{2}{*}{ Outcome } & \multicolumn{2}{c}{ Training set } \\
\cline { 2 - 3 } $0(-)$ life & $0(-)$ life & $1(+)$ death \\
$1(+)$ death & 4178 & 6 \\
\hline
\end{tabular}

Table 3. The confusion matrix for the test set for C5.0 algorithm.

\begin{tabular}{lcc}
\hline \multirow{2}{*}{ Outcome } & \multicolumn{2}{c}{ Test set } \\
\cline { 2 - 3 } $0(-)$ life & $0(-)$ life & $1(+)$ death \\
$1(+)$ death & 2113 & 3 \\
\hline
\end{tabular}

Table 4. The confusion matrix for the validation set for C5.0 algorithm.

\begin{tabular}{lcc}
\hline \multirow{2}{*}{ Outcome } & \multicolumn{2}{c}{ Validation set } \\
\cline { 2 - 3 } & $0(-)$ life & $1(+)$ death \\
\hline $0(-)$ life & 2111 & 5 \\
$1(+)$ death & 17 & 9 \\
\hline
\end{tabular}

Table 5. Ruleset for C5.0 algorithm.

\begin{tabular}{|c|c|}
\hline IF & THEN \\
\hline $\begin{array}{l}\mathrm{x} 3<=8.203 \text { and } \mathrm{x} 27 \text { in }\left[\begin{array}{lllll}0 & 1 & 2 & 3 & 4\end{array}\right] \text { and } \mathrm{x} 71=1 \\
\text { and } \mathrm{x} 9>8.755\end{array}$ & life \\
\hline $\begin{array}{l}x 3<=8.203 \text { and } x 27 \text { in }\left[\begin{array}{lllll}0 & 1 & 2 & 3 & 4\end{array}\right] \text { and } x 71 \text { in } \\
{\left[\begin{array}{lll}2 & 4\end{array}\right]}\end{array}$ & life \\
\hline $\begin{array}{l}x 3<=8.203 \text { and } x 27=5 \text { and } x 26 \text { in }\left[\begin{array}{ll}12 & 25 \\
28 & 31\end{array}\right]\end{array}$ & life \\
\hline $\mathrm{x} 3>8.203$ & life \\
\hline $\begin{array}{l}\mathrm{x} 3<=8.203 \text { and } x 27 \text { in }\left[\begin{array}{lllll}0 & 1 & 2 & 3 & 4\end{array}\right] \text { and } x 71=1 \\
\text { and } x 9<=8.755\end{array}$ & death \\
\hline $\begin{array}{l}x 3<=8.203 \text { and } x 27 \text { in }\left[\begin{array}{lllll}0 & 1 & 2 & 3 & 4\end{array}\right] \text { and } x 71 \text { in } \\
{\left[\begin{array}{lll}3 & 6\end{array}\right]}\end{array}$ & death \\
\hline $\mathrm{x} 3<=8.203$ and $\mathrm{x} 27=5$ and $\mathrm{x} 26$ in $\left[\begin{array}{ll}15 & 24\end{array}\right]$ & death \\
\hline
\end{tabular}


Also the CHAID tree may be converted into set of rules which are listed in Table 6. In each rule assigned the major classification of the corresponding node. Rules for 0 (life) contains 14 rules.

Finally, we evaluated the performance of the aforementioned classification algorithms by means of ROC curves methodology, complemented by determination of the areas under the curves, as presented in Table 7.

We observe from the results derived from ROC curves methodology that CHAID algorithm has the biggest AUC $=0.888$ which indicates an excellent performance of classifiers and a very good discriminating ability about the patient's outcome (life or death). C5.0 algorithm has the biggest value for the overall accuracy and a very satisfactory AUC $=0.709$ which indicates a good performance of classifiers and a satisfactory discriminating ability about the patient's outcome. The C\&RT algorithm has AUC $=0.5$ which indicates a random performance of classifiers and an unreasonable discriminating ability to diagnose patients with and without the disease/condition. It is therefore natural not to trust the results of C\&RT algorithm, as expected, from our previous effort to build a decision tree and a ruleset for C\&RT where we observed that there were no clear results from the Clementine because it could not generate a rule set (conditions too complex). Generally, Classification algorithms were successful on trauma data set. The classification accuracy was especially high, reaching accuracy of $99 \%$ of correct classifications.

In Figure 1 we present the evaluation of testing set for all the classification algorithms by means of ROC curves.

Table 6. Ruleset for CHAID algorithm.

\begin{tabular}{|c|c|}
\hline IF & THEN \\
\hline $\mathrm{x} 71=1$ and $\mathrm{x} 3<=13.081$ & life \\
\hline $\mathrm{x} 71=1$ and $\mathrm{x} 3>13.081$ & life \\
\hline$x 71=2$ and $x 19<=3$ and $x 50=0$ & life \\
\hline$x 71=2$ and $x 19<=3$ and $x 50=1$ & life \\
\hline $\begin{array}{l}x 71=2 \text { and } x 19>3 \text { and } x 19<=3.945 \\
\text { and } x 50=0\end{array}$ & life \\
\hline $\begin{array}{l}x 71=2 \text { and } x 19>3 \text { and } x 19<=3.945 \\
\text { and } x 50=1\end{array}$ & life \\
\hline$x 71=2$ and $x 19>3.945$ and $x 19<=4$ & life \\
\hline $\begin{array}{l}x 71=2 \text { and } x 19>4 \text { and } x 19<=4.888 \text { and } \\
x 11<=17827.408\end{array}$ & life \\
\hline $\begin{array}{l}\mathrm{x} 71=2 \text { and } \mathrm{x} 19>4 \text { and } \mathrm{x} 19<=4.888 \text { and } \\
\mathrm{x} 11>17827.408\end{array}$ & life \\
\hline $\mathrm{x} 71=2$ and $\mathrm{x} 19>4.888$ & life \\
\hline $\mathrm{x} 71=3$ or $\mathrm{x} 71=4$ & life \\
\hline $\mathrm{x} 71=6$ and $\mathrm{x} 3<=11.447$ & life \\
\hline$x 71=6$ and $x 3>11.447$ and $x 28=0$ & life \\
\hline $\mathrm{x} 71=6$ and $\mathrm{x} 3>11.447$ and $\mathrm{x} 28=1$ & life \\
\hline
\end{tabular}

At clustering we specified as minimum number of clusters 2 and as maximum number of clusters 15 where we received detailed clustering of the records (distribution of variables with a percentage $>85 \%$ for its values). The clusters are obtained automatically from the performance of TwoStep algorithm, using only the most significant fields of the Trauma data set as they have been derived from feature selection algorithm. The number of clusters was 5 grouping 336, 914, 1227, 1050, 729 number of records.

For the clustering analysis, we performed additionally the K-means algorithm and we determined 5 clusters as default so that records within a cluster are similar to each other and distinct from records in other clusters, where we also received detailed clustering of the records. Five clusters are obtained from the performance of K-means algorithm, using only the most significant fields of the Trauma data set as they have been derived from feature selection algorithm. Each cluster contained 1280, 1010, 379, 935, 652 records.

Hence, we achieved the first goal of clustering, that is the decomposition of the data set into categories of similar data. Clusters are defined by their centers, where a cluster center is a vector of values for the input fields. Results deriving from clustering analysis, are reported as following: for discrete fields the mean value for training records assigned to each cluster is presented. For continuous fields we present only the major value of the variable, the major percentage which belongs to each cluster. Both algorithms gave identical rules. The TwoStep created the most multitudinous cluster containing

Table 7. Performance of the classification algorithms.

\begin{tabular}{lcc}
\hline \multirow{2}{*}{$\begin{array}{c}\text { Algo- } \\
\text { rithm }\end{array}$} & \multicolumn{2}{c}{ Area Under the Curve } \\
\cline { 2 - 3 } & Overall accuracy & AUC \\
\hline CHAID & 98.602 & 0.888 \\
C5.0 & 98.835 & 0.709 \\
C\&RT & 98.602 & 0.500 \\
\hline
\end{tabular}

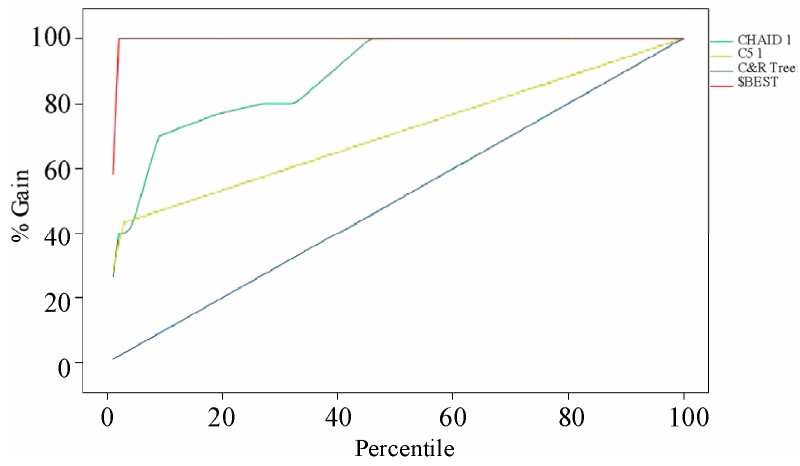

Figure 1. Evaluation of Testing set for CHAID, C5.0, C\& RT algorithms. 
1227 cases which were middle aged (50 years old on average, (o.a)), weighted $73.2 \mathrm{~kg}$ (o.a), had white cells 10537 (o.a), glucose levels 125 (o.a), creatinine 1.04 (o.a), urea 40 (o.a), good evaluation of disability (4.3, o.a), not severely injured (Injury Severity Score mean=6.2), high GCS (14.76, (o.a)), 84 pulses (o.a), systolic arterial pressure 130.5 (o.a), diastolic arterial pressure 77.5 (o.a), Ht 40 (o.a), Hb 13.4 (o.a). Additionally these patients were not pale (96.4\%), had not ephidrosis (97.3\%), had hydration with fluids (88.8\%), had done radiography and CT (92.1\%, 89.5\% respectively), and were admitted to hospital clinic after the Emergency Room treatment (90.6\%).

Using association rules, we performed the Generalized Rule Induction (GRI) algorithm in order to summarize patterns in the data using a quantitative measure for the interestingness of rules. The consequent (the "then" part of the rule) is restricted to being a single value assignment expression ( $\mathrm{Y}=1$ death) while the antecedent (the "if" part of the rule) may be a conjunction of expressions of only the most significant fields of the Trauma data set as they have been derived from feature selection algorithm. Each rule in the final ruleset has associated support, confidence, based on the number of records for which the antecedent and the entire rule are true. Defining minimum antecedent support equal to $0 \%$, minimum rule confidence equal to $50 \%$, maximum number of antecedents equal to 3 , maximum number of rules equal to 100 and choosing only true values for flags, resulted in the appearance only of the set of rules with consequent $y=1$ death. Four association rules are obtained from the performance of GRI algorithm and this set of association rules is presented in Table $\mathbf{8}$.

According to the results derived from the implementation of GRI association rule, mortality is predicted with higher percent of support (1.03\%) and confidence 60\% when G.C.S (x3) is smaller than 6.

Moreover the model suggests with the highest confidence that people with a cutpoint of G.C.S under 6 although that are transferred to hospital with ambulance and they don't suffer from lower limbs injury they are predicted to die (Support: 0.96\%, Confidence: 63.41\%).

\section{CONCLUSIONS}

In conclusion, the selection of the most important factors

Table 8. Ruleset for GRI algorithm.

\begin{tabular}{ll}
\hline \multicolumn{1}{c}{ IF } & THEN \\
\hline $\mathrm{x} 3<5.97405$ and $\mathrm{x} 25=1$ & death \\
and $\mathrm{x} 108=0$ & death \\
$\mathrm{x} 3<5.97405$ and $\mathrm{x} 108=0$ & death \\
$\mathrm{x} 3<5.97405$ and $\mathrm{x} 25=1$ & death \\
$\mathrm{x} 3<5.97405$ & \\
\hline
\end{tabular}

determining the outcome of injured patients is critical, particularly when the problem is high dimensional. Therefore in order to detect the requested information, it is imperative to use expertise and cutting-edge statistical methods that would meet these needs. Data mining can be considered as an in-depth research to find information previously not seen in many of the collected data and has recently been used to medical data [16] often giving useful information for patterns. In our study, the results were encouraging because the implemented algorithms generated useful rules that are logical, consistent with the medical experience and provide more specific information which may assist as guidelines for trauma management. Specifically, we found that the CHAID and C5.0 algorithms offer an extensive knowledge of the classification of injuries including combinations of features that lead to death or good outcome. Also the K-mean and TwoStep algorithms produce casualties with common features and the classification is particularly interesting in the latter case where groups are not determined by the analyst. The comparison of data mining methods in terms of evaluation of medical diagnostic procedures for sensitivity, specificity, Positive Predictive value, Negative Predictive Value, confirmed that the extraction of data from a medical basis as this, may contribute to detect factors or combinations of factors that can predict reliably trauma patients outcome.

\section{REFERENCES}

[1] The trauma audit and research network. http://www. tarn.ac.uk/introduction/firstDecade.pdf

[2] Meyer, A. (1998) Death and disability from injury: A global challenge. Journal of Trauma, 44(1), 1-12.

[3] World Health Organization. http://www.who.int/en/

[4] The trauma audit and research network. http://www. tarn.ac.uk/content/downloads/36/firstdecade.pdf

[5] Baker, P., O’Neil, B., Haddon, W. and Long, B. (1974) The injury severity score: A method for describing patients with multiple injuries and evaluating emergency care. Journal of Trauma, 14(3), 187-196.

[6] Copes, W.S., Sacco, W.J., Champion, H.R. and Bain, L.W. (1990) Progress in characterising anatomic injury. Proceedings of the 33rd Annual Meeting of the Association for the Advancement of Automotive Medicine, Baltimore, 2-4 October 1989, 205-218.

[7] Teasdale, G. and Jennett, B. (1974) Assessment of coma and impaired consciousness. A practical scale. Lancet, 2(7872), 81-84.

[8] Penny, K. and Chesney, T. (2006) Imputation methods to deal with missing values when data mining trauma injury data. Proceedings of 28th International Conference on Information Technology Interfaces, Cavtat, 19-22 June 2006, 213-218.

[9] Donders, A.R., Van der Heijden, G.J., Stijnen, T. and Moons, K.G. (2006) Review: A gentle introduction to imputation of missing values. Journal of Clinical Epi- 
demiology, 59(10), 1087-1091.

[10] Cox, D.R. and Hinkley, D.V. (1974) Theoretical statistics. Chapman and Hall, London.

[11] Cramer, H. (1946) Mathematical methods of statistics. Princeton University Press, Princeton.

[12] Dobson, A. (2002) An introduction to generalized linear models. 2nd Edition, Chapman and Hall/CRC, London.

[13] Pearson, R.L. (1983) Karl Pearson and the chi-squared test. International Statistical Review, 51, 59-72.

[14] Agrawal, R. and Srikant, R. (1994) Fast algorithms for mining association rules. Proceedings of the 20th International Conference on Very Large Databases, Santiago de Chile, 12-15 September 1994, 479-499.

[15] Craven, P. and Wahba, G. (1979) Smoothing noisy data with spline functions: Estimating the correct degree of smoothing by the method of generalized cross-validation. Numerische Mathematik, 31, 377-403.

[16] Breault, J.L., Goodall, C.R. and Fos, P.J. (2002) Data mining a diabetic data warehouse. Artificial Intelligence in Medicine, 26(1-2), 37-54. 